\title{
Estudo retrospectivo de melanomas cutâneos e mucosos na população do estado do Rio Grande do Norte, Brasil
}

\author{
Retrospective study of cutaneous and mucosal melanomas in the population of the state of \\ Rio Grande do Norte, Brazil
}

Rafael Lopes Ferreira Lima'; Marília Queiroga Rocha Nóbrega'; Maria de Lourdes Silva Arruda de Moraes²; Márcia Cristina da Costa Miguel33; Kenio Costa Lima ${ }^{4}$, Éricka Janine Dantas da Silveira ${ }^{5}$

\section{unitermos \\ Melanomas cutâneos \\ Melanomas mucosos \\ Epidemiologia}

\section{resumo}

Introdução e objetivos: O melanoma é uma neoplasia maligna que pode acometer a pele e as mucosas. A taxa de incidência do melanoma cutâneo de forma geral tem aumentado nos últimos anos. Esta pesquisa realizou uma análise retrospectiva de melanomas cutâneos e mucosos no período de 30 anos em um serviço do Rio Grande do Norte, enfatizando características clínicas, conduta e evolução dos melanomas mucosos orais. Material e método: Nos prontuários clínicos, foram coletadas informações sobre idade, raça, sexo, localização o tipo de comprometimento da lesão, se primária ou metastática. Resultados: Um total de 397 casos de melanoma foi diagnosticado, sendo 332 cutâneos $(83,6 \%$ ) e 46 mucosos $(11,6 \%)$. O tronco foi mais acometido $(27,7 \%)$, seguido por membros inferiores $(24,7 \%)$ e cabeça e pescoço (18,9\%). Entre os melanomas mucosos, a maioria era de cabeça e pescoço $(76,1 \%)$. De forma geral, $52,4 \%$ ocorreram em indivíduos do sexo masculino e $47,6 \%$, no feminino. A idade média foi de 54,9 anos. Nos homens esteve mais presente nas sétima e oitava décadas de vida, e nas mulheres, nas quinta e sexta décadas. Seis casos $(1,5 \%)$ acometeram a mucosa oral e estes ocorreram mais em indivíduos do sexo feminino, acima de 55 anos. Conclusão: $O$ perfil epidemiológico dos casos de melanomas avaliados se assemelha aos poucos estudos epidemiológicos publicados, apesar de alguns dados, no tocante a sexo e idade em determinadas localizações anatômicas, terem variado e a incidência de melanomas orais ter sido bem mais baixa do que a relatada na maioria das pesquisas.

\section{abstract}

Introduction and objectives: Melanoma is a malignant neoplasm that occurs in the skin and mucosae. Overall, the incidence rate of cutaneous melanoma has increased in the last years. It was performed a retrospective analysis of cutaneous and mucosal melanomas diagnosed within a period of 30 years in one health care institution of Rio Grande do Norte, highlighting clinical features, approach and evolution of oral mucosal melanomas. Methods: Data as to age, race, gender, tumor topography and lesion involvement (metastatic or primary) were collected from clinical records. Results: A total of 397 cases of melanoma were diagnosed, 332 of them were cutaneous (83.6\%) and 46 of them were mucosal (11.6\%). The trunk was the most affected area (27.7\%), followed by lower limbs (24.7\%) and head/neck (18.9\%). Within the mucosal melanomas, most cases were on head and neck (76.1\%). By and large, $52.4 \%$ affected male individuals and $47.6 \%$ affected female patients. The mean age was 54.9. Among men it was more prevalent in the $7^{\text {th }}$ and $8^{\text {th }}$ decades of life, whereas among women it was more prevalent in the $5^{\text {th }}$ and $6^{\text {th }}$ decades of life. $A$ total of 6 cases (1.5\%) occurred in the oral mucosa, with more prevalence among female individuals over 55 years of age. Conclusions: The epidemiological profile of the analyzed cases of melanoma was similar to the few published epidemiological studies, although some data on gender and age in certain anatomical sites varied and the incidence of oral melanoma was much lower than that reported in most investigations. key words

Cutaneous melanomas

Mucosal melanomas

Epidemiology

1. Cirurgião-dentista do Departamento de Odontologia da Universidade Federal do Rio Crande no Norte (UFRN).

2. Mestra em Patologia Oral pelo Programa de Pós-Graduação em Patologia Oral da UFRN; professora assistente de Patologia Geral e Oral da Universidade Estadual do Rio Crande no Norte (UERN); cirurgiã-dentista do Hospital Dr. Luiz Antônio.

3. Doutora em Patologia Oral; professora adjunta II DE do Departamento de Odontologia da UFRN.

4. Doutor em Microbiologia pela Universidade Federal do Rio de Janeiro (UFR)); professor adjunto IV DE do Departamento de Odontologia da UFRN.

5. Doutora em Patologia Oral; professora adjunta da UFRN. 


\section{Introdução}

O melanoma é uma neoplasia maligna que, na maioria dos casos, origina-se da transformação maligna de melanócitos da pele e/ou da mucosa. Apenas 1,3\% dos melanomas acomete as mucosas e, destes, cerca de $55 \%$ ocorrem na região de cabeça e pescoço, $18 \%$ no trato genital feminino, $23,8 \%$ na região anal/retal e 2,8\% no trato urinário ${ }^{(3)}$. Em relação à região cervicofacial, o local mais comum para o aparecimento do melanoma é a conjuntiva, seguido pelas vias aéreas superiores e mucosa oral(11).

Apesar da ocorrência comum do melanoma cutâneo, o das mucosas é uma entidade muito rara, compreendendo de $0,8 \%$ a $3,7 \%$ de todos os melanomas ${ }^{(1)}$. Destes, o melanoma oral primário é um tumor extremamente raro e agressivo que surge na mucosa da boca, representando de $0,2 \%$ a $8 \%$ de todos os melanomas na Europa e nos EUA e somente $0,5 \%$ de todas as lesões malignas orais $(3,5)$.

Os sítios intraorais mais acometidos pelo melanoma são o palato e a gengiva maxilar. Porém a gengiva mandibular, a mucosa jugal, os lábios e a gengiva alveolar são outros locais que podem ser acometidos ${ }^{(5,6)}$. O melanoma oral primário pode ocorrer em qualquer idade, mas é extremamente raro em pessoas com menos de 30 anos, sendo a idade média de 55 anos. Nesse local, o melanoma exibe predileção pelo sexo masculino ${ }^{(11)}$.

De acordo com Meleti et al.(5), o prognóstico do melanoma oral é mais pobre do que o dos cutâneos, pois apenas 15\% dos pacientes sobrevivem em cinco anos. Ainda é desconhecido se o pior prognóstico do melanoma oral, quando em comparação com o cutâneo, deve-se às diferenças em suas características histológicas, ao seu diagnóstico tardio ou se as suas características anatômicas são determinantes para tal diferença ${ }^{(2)}$. O melanoma oral exibe tendência para recorrer localmente e desenvolver metástases a distância(1).

Assim, o objetivo desse estudo consistiu em analisar o perfil da população do estado do Rio Grande do Norte/Brasil acometida pelos melanomas cutâneos e mucosos em um período de 30 anos, comparando-os com os dados da literatura mundial, com destaque para os casos de melanoma oral primário em decorrência do seu comportamento clínico mais agressivo.

\section{Materiais e métodos}

O presente estudo realizou uma análise retrospectiva de todos os casos de melanomas cutâneos e mucosos diagnosticados no Hospital Dr. Luiz Antônio, em Natal-RN (serviço de referência do estado), em um período de 30 anos, com a finalidade de analisar o perfil da população rio-grandense-do-norte acometida por essas lesões.

A partir dos prontuários clínicos constantes no setor de registro de câncer do referido serviço foram coletadas as informações referentes a idade, raça, sexo dos pacientes, topografia do tumor, bem como o tipo de comprometimento da lesão, se primária ou metastática. Quanto à localização, os melanomas foram considerados difusos quando presentes em vários locais do organismo, e múltiplos quando havia várias lesões próximas umas das outras. Com os dados obtidos foi realizada uma análise descritiva, estabelecendo-se valores médios e percentuais. Toda a análise dos dados foi realizada pelo software SPSS 13.0 for Windows. Este estudo foi aprovado pelo Comitê de Ética em Pesquisa do Hospital Dr. Luiz Antônio (Parecer no 008/0008/2007).

\section{Resultados}

Um total de 397 casos de melanoma foi diagnosticado no serviço pesquisado no período de 30 anos, sendo 332 destes do tipo cutâneo $(83,6 \%)$ e 46 do tipo mucoso $(11,6 \%)$. Em 19 casos (4,8\%) não havia essa informação.

De forma geral, a distribuição de todos os casos de melanomas encontra-se listada na Tabela 1. O tronco foi a região mais acometida $(110 / 27,7 \%)$, seguido por mem-

\begin{tabular}{|c|c|c|}
\hline \multirow{2}{*}{$\frac{\text { Tabela } 1}{\text { Localização }}$} & \multicolumn{2}{|c|}{$\begin{array}{l}\text { Distribuição dos casos de } \\
\text { melanoma quanto à localização }\end{array}$} \\
\hline & $n$ & Porcentagem (\%) \\
\hline Tronco & 110 & 27,7 \\
\hline Membro inferior & 98 & 24,7 \\
\hline $\begin{array}{l}\text { Cabeça e } \\
\text { pescoço }\end{array}$ & 81 & 20,4 \\
\hline $\begin{array}{l}\text { Membro } \\
\text { superior }\end{array}$ & 31 & 7,8 \\
\hline Difuso & 18 & 4,5 \\
\hline Região axilar & 13 & 3,3 \\
\hline Pele SOE & 12 & 3 \\
\hline Múltiplo & 7 & 1,8 \\
\hline Região inguinal & 6 & 1,5 \\
\hline$S / l$ & 21 & 5,3 \\
\hline Total & 397 & 100 \\
\hline
\end{tabular}

SOE: sem outra especificação; S/l: sem informação. 
bros inferiores (98/24,7\%) e cabeça e pescoço (81/20,4\%). Entre os melanomas mucosos, merecem destaque, devido a diferenças no comportamento biológico, seis casos em cavidade oral (1,5\%).

Entre os melanomas mucosos, os mais comuns foram os de cabeça e pescoço $(76,1 \%)$, seguidos pelos de trato genital feminino $(13,1 \%)$, trato urinário-retal $(6,5 \%)$ e trato gastrointestinal (4.3\%) (Figura 1). Dos 35 casos localizados em cabeça e pescoço, 21 acometeram o globo ocular (60\%); seis, as vias aéreas superiores $(17,15 \%)$; seis, a cavidade oral (17,15\%); e dois, a parótida (5,7\%) (Figura 2).

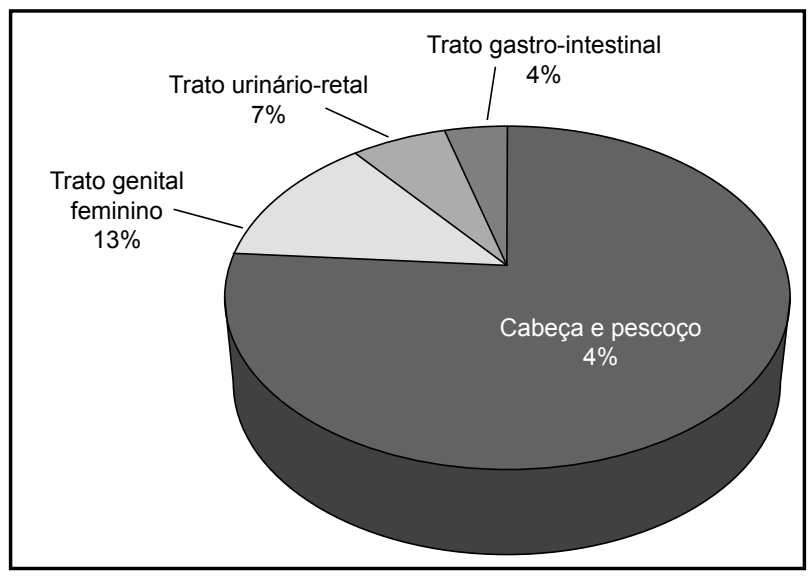

Figura 1 - Distribuição dos casos de melanomas mucosos de acordo com a localização

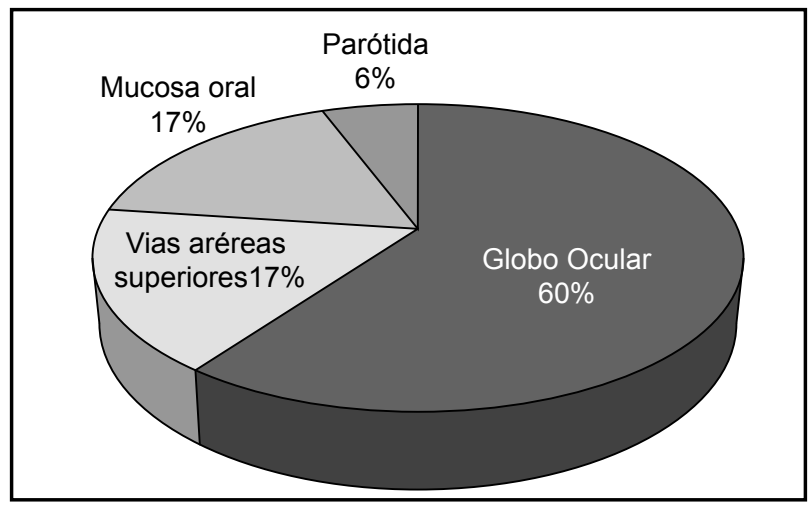

Figura 2 - Distribuição dos casos de melanomas mucosos de cabeça e pescoço de acordo com a localização

Quanto ao sexo, entre todos os tipos de melanomas, $52,4 \%$ ocorreram em homens e $47,6 \%$ em mulheres. Indivíduos da raça parda foram mais acometidos $(41,1 \%)$, seguidos por aqueles de raças branca $(26,4 \%)$, negra $(9,8 \%)$ e amarela (4,8\%). Em $71(17,2 \%)$ casos não havia essa informação na ficha clínica.

Os pacientes apresentaram idade média de 54,9 anos, variando de 5 a 98 anos. Não houve diferença entre aqueles com idade inferior a 55 anos $(49,6 \%)$ e os com 55 anos ou mais $(50,4 \%)$. Os indivíduos do sexo masculino apresen- taram média de idade de 57,4 anos, enquanto os do feminino, de 52,1 anos. Além disso, nos homens, o melanoma esteve mais presente na sétima e oitava décadas de vida, enquanto nas mulheres a quinta e sexta décadas foram as mais acometidas (Figura 3).

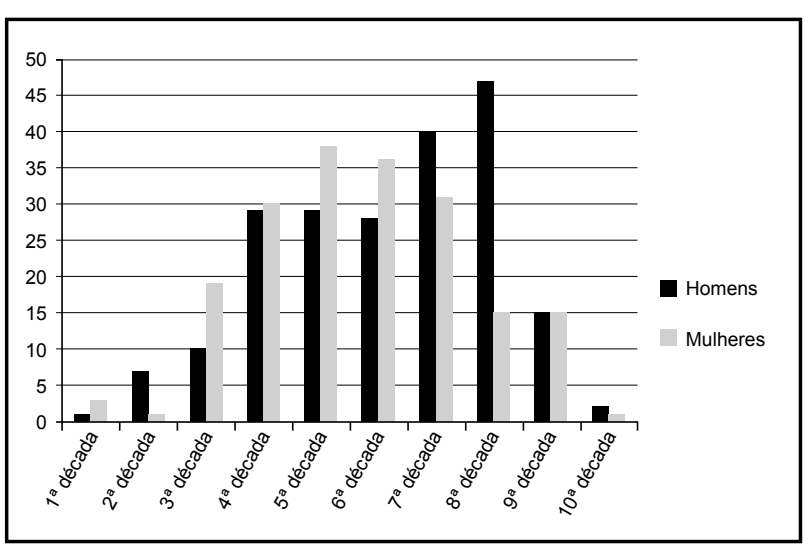

Figura 3 - Distribuição dos casos de melanomas de acordo com a faixa etária dos pacientes e o sexo

Houve predileção pelo sexo masculino nos casos de região axilar $(61,5 \%)$, tronco $(59,1 \%)$, cabeça e pescoço (58\%), forma múltipla $(57,1 \%)$ e membro inferior $(52 \%)$. As mulheres foram mais acometidas pelos melanomas localizados em pele sem outra especificação (SOE) (75\%), membro superior $(71 \%)$, região inguinal $(66,7 \%)$ e de forma difusa (66,7\%) (Figura 4). A informação quanto a se o melanoma era primário ou metastático esteve ausente na maioria das fichas clínicas (63,2\%). Entretanto, nos casos que continham tal informação, não houve grande diferença entre os casos locais (44 casos), as metástases regionais (48 casos) e as metástases a distância (54 casos).

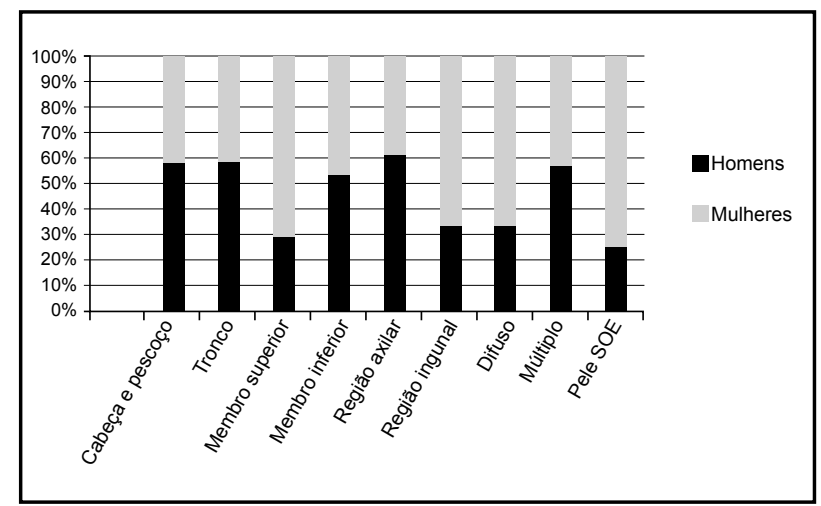

Figura 4 - Localização dos melanomas distribuídos de acordo com o sexo dos indivíduos

De todas as regiões acometidas pelos melanomas, o membro inferior e a região de cabeça e pescoço foram as únicas de ocorrência mais comum em indivíduos com mais de 55 anos (Figura 5). 


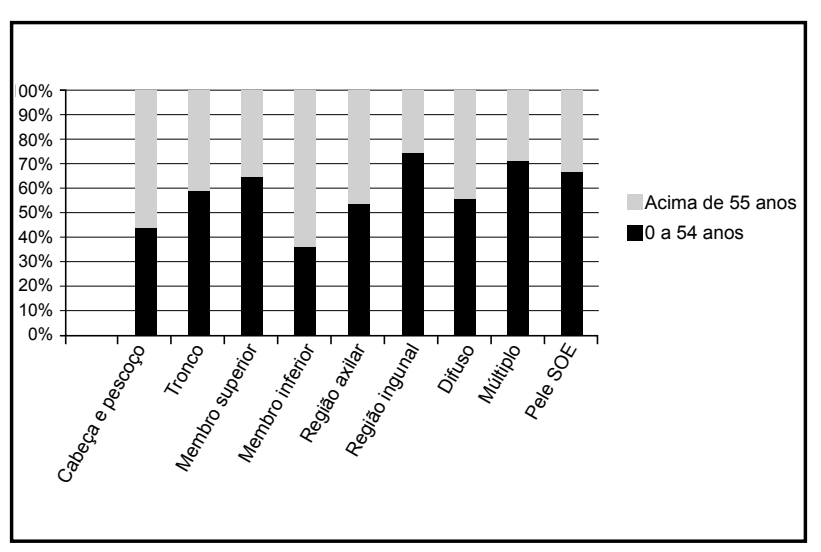

Figura 5 - Localização dos melanomas distribuídos de acordo com a idade dos indivíduos

No tocante a informações clínicas, tratamento e evolução dos casos de melanoma mucoso oral, verificou-se que os mesmos estiveram mais presentes no sexo feminino, no palato duro e que, em todos os casos, os pacientes foram a óbito em pouco tempo (Tabela 2).

\section{Discussão}

A presente pesquisa analisou o perfil de pacientes acometidos por melanomas cutâneos e mucosos no período de 30 anos em um serviço de referência do estado Rio Grande do Norte, delineando uma análise comparativa dos dados obtidos nessa população com os constantes na literatura mundial. Esse estado exibe uma temperatura média anual em torno de $25,5^{\circ} \mathrm{C}$, com máxima de $31,3^{\circ} \mathrm{C}$ e mínima de $21,1^{\circ} \mathrm{C}$, e predomínio de dias ensolarados na maior parte do ano ${ }^{(10)}$, predispondo a população a acentuada exposição solar, o que é um fator de risco bem estabelecido para o surgimento dos melanomas cutâneos ${ }^{(9)}$.

Em uma escala global, o melanoma de pele é estimado como sendo o $16^{\circ}$ e 15 - diagnosticado mais comumente em homens e mulheres, respectivamente ${ }^{(12)}$, e o melanoma oral é extremamente raro, correspondendo de 0,2\% a $0,8 \%$ de todos os melanomas( ${ }^{(5)}$. Em nosso estudo, os

\section{Sexo, idade, localização, características clínicas e tratamento dos casos de melanoma localizados}

Tabela 2 na mucosa oral

\begin{tabular}{|c|c|c|c|c|c|c|}
\hline Caso & Sexo & $\begin{array}{l}\text { Idade } \\
\text { (anos) }\end{array}$ & Localização & Características clínicas & Tratamento & Sobrevida \\
\hline 1 & M & 72 & Palato duro & $\begin{array}{l}\text { Lesão proliferativa, nodular, } \\
\text { pigmentada, crescimento vertical } \\
\text { com invasão das fossas nasais e } \\
\text { seios maxilar e esfenoidal }\end{array}$ & IT e RT & 10 meses \\
\hline 2 & $\mathrm{~F}$ & 76 & $\begin{array}{l}\text { Lábio } \\
\text { superior }\end{array}$ & $\begin{array}{l}\text { Lesão macular, pigmentada, } 1,5 \mathrm{~cm} \\
\text { de diâmetro, crescimento vertical, } \\
\text { bases e margens livres }\end{array}$ & $\begin{array}{c}\text { Ressecção da } \\
\text { lesão }\end{array}$ & Desc. \\
\hline 3 & $\mathrm{~F}$ & 51 & Palato duro & $\begin{array}{l}\text { Lesão macular, azulada, irregular, } \\
\text { ulceração central, crescimento } \\
\text { vertical, } 2,5 \mathrm{~cm} \text { de diâmetro }\end{array}$ & IT e RT & 12 meses \\
\hline 4 & $F$ & 65 & Palato duro & $\begin{array}{l}\text { Lesão macular, pigmentada, } \\
\text { crescimento vertical, limites } \\
\text { imprecisos, bordas irregulares }\end{array}$ & $\begin{array}{l}\text { Nenhum (foi a } \\
\text { óbito antes do } \\
\text { tratamento) }\end{array}$ & 4 dias \\
\hline 5 & M & 80 & Palato duro & $\begin{array}{l}\text { Lesão proliferativa, nodular, } \\
\text { invasão da mucosa jugal, vascular, } \\
\text { óssea, perineural e do seio maxilar }\end{array}$ & $\begin{array}{c}\text { Ressecção da } \\
\text { hemimaxila }\end{array}$ & 11 meses \\
\hline 6 & $\mathrm{~F}$ & 59 & Palato duro & $\begin{array}{l}\text { Lesão macular, pigmentada, com } \\
\text { manchas hipercrômicas, irregular, } \\
\text { sangrante, } 1,5 \mathrm{~cm} \text { de diâmetro, } \\
\text { invasão de palato mole e pilares } \\
\text { faríngeos }\end{array}$ & $\begin{array}{c}\text { Ressecção da } \\
\text { lesão e IT }\end{array}$ & 6 meses \\
\hline
\end{tabular}

M: masculino; F: feminino; IT: imunoterapia; RT: radioterapia. 
melanomas cutâneos corresponderam a $83,6 \%$ da amostra, enquanto os mucosos e orais compreenderam 10,1\% e $1,5 \%$, respectivamente, fatos coerentes com os referidos pela literatura mundial.

Pruthi et al. ${ }^{(9)}$ verificaram, em seu estudo, um aumento na incidência de melanomas cutâneos nos últimos anos, principalmente em pacientes mais jovens, relatando que as localizações anatômicas mais acometidas também estão mudando, já que no período de 1956 a 1980 a cabeça e o pescoço eram os sítios mais comprometidos, seguidos pelo tronco. Mas nos últimos 25 anos, o tronco passou a ser o local mais acometido na população estudada. Nosso estudo concorda com os últimos resultados da referida pesquisa, já que o tronco foi o local mais acometido pelo melanoma cutâneo com $27,7 \%$, seguido pelos membros inferiores. Ainda na mesma pesquisa, a média de idade para os pacientes dos sexos masculino e feminino foi de 58,7 e 54,3 anos, respectivamente. Tais achados foram bem semeIhantes aos nossos, já que a média de idade para os homens foi de 57,4 anos e de 52,1 anos para as mulheres. Neste mesmo estudo, foi encontrado aumento da frequência de melanomas cutâneos em pacientes jovens ( $<40$ anos). Os autores atribuem tal fato à possibilidade da presença de alterações genéticas nesses pacientes.

Em nosso estudo, houve frequência semelhante entre os sexos acometidos pelos melanomas cutâneos, diferente do que foi observado na pesquisa de Lasithiotakis et al. ${ }^{(4)}$, que verificaram um índice $30 \%$ maior em mulheres. Neste mesmo estudo foi verificada redução na mortalidade em ambos os sexos no período de análise de 28 anos. Segundo esses autores, isso ocorreu devido provavelmente à melhora na consciência pública em relação às lesões pigmentadas suspeitas e ao diagnóstico precoce desses tumores.

Pruthi et al. ${ }^{(9)}$ verificaram diminuição na frequência de melanomas cutâneos em mulheres com menos de 60 anos e isso, segundo os autores, sugere que comportamentos de proteção contra o sol estão sendo mais adotados e que mudanças diretas ou indiretas no estilo de vida, como, por exemplo, o aumento do ingresso de mulheres no mercado de trabalho, estão gerando efeitos positivos.

Em relação aos fatores de risco envolvidos no surgimento dos melanomas, Sortino-Rochou et al. ${ }^{(12)}$ reforçam que tanto o envolvimento familiar como fatores ambientais podem contribuir para o seu surgimento. Esses mesmos autores ressaltam que a presença de nevos displásicos, pele clara e exposição ao sol parece estar mais relacionada com o surgimento de melanomas cutâneos, bem como com problemas no reparo do DNA. Deficiência nos mecanismos de reparo celular pode explicar o aparecimento dessa neoplasia em pessoas que não se expõem substancialmente ao sol. Em nossa pesquisa, não foi possível estabelecer a associação entre os fatores de risco e o surgimento de melanomas cutâneos, haja vista não haver tal informação nos prontuários analisados. Entretanto, os indivíduos melanodermas foram os menos acometidos por esses tumores.

Em relação aos melanomas mucosos, Patrick et al. ${ }^{(7)}$ relataram que os mesmos são extremamente raros $(0,03 \%$ de todos os cânceres) e exibem pobre prognóstico por serem mais agressivos quando comparados com os cutâneos, ressaltando que, diferente dos melanomas cutâneos que vêm exibindo aumento na incidência, a frequência dos mucosos tem permanecido relativamente estável nos últimos anos. Segundo esses autores, enquanto os melanomas cutâneos compreendem $91,2 \%$ de todos os melanomas, o ocular e o de mucosas são responsáveis por $5,2 \%$ e 1,3\%, respectivamente. Quanto aos melanomas mucosos, é relatado por tais autores que os localizados em cabeça e pescoço são os mais comuns $(55,4 \%)$, seguidos pelos de trato genital feminino, tratos anal e retal e urinário. Esses dados são semelhantes aos da nossa pesquisa, que revelou uma porcentagem de $83,6 \%$ para os melanomas cutâneos e $11,6 \%$ para todos os melanomas mucosos, sendo, destes, os mais comuns localizados em cabeça e pescoço, seguidos pelos de trato genital feminino, trato urinário-retal e gastrointestinal.

Sobre os melanomas mucosos, Patrick et al..$^{(7)}$ relataram que os de cavidade oral compreendem cerca de $40 \%$ dos casos, comentando que os mesmos exibem tendência a ocorrer em pacientes com menos de 40 anos de idade, sendo o palato duro e o rebordo alveolar os sítios mais acometidos. Diferentemente desses achados, os melanomas orais do nosso estudo ocorreram mais em mulheres acima de 55 anos, porém, de forma semelhante, foram mais frequentes no palato duro, sendo a localização intraoral

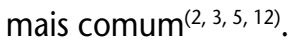

Em pesquisa realizada por Bachar et al.(1), os melanomas mucosos orais foram encontrados em $10(16,4 \%)$ dos 61 casos de mucosos de cabeça e pescoço estudados, enquanto em nossa pesquisa os referidos casos corresponderam a apenas $1,5 \%$ da amostra total e a $17 \%$ entre os melanomas mucosos, o que expressa, segundo esses autores, a raridade dos melanomas orais e a necessidade de cooperação multicêntrica para o correto estabelecimento de conduta clínica e estadiamento para os melanomas mucosos orais.

No tocante ao sexo dos pacientes acometidos por melanomas mucosos orais, Sortino-Rachou et al. ${ }^{(12)}$ reportam que alguns estudos citam que os homens são mais acometidos 
por esse tipo de melanoma e, em outros, as mulheres. Na presente pesquisa, as mulheres foram as mais acometidas pelos melanomas orais.

Conforme relatos de Pour ${ }^{(8)}$, nenhum fator etiológico tem sido identificado para os melanomas orais, ressaltando que alguns parecem surgir de um nevo preexistente e outros, "de novo". Esses mesmos autores citaram que, clinicamente, esses tumores são classificados em cinco tipos: nodular pigmentado, nodular não pigmentado, macular pigmentado, misto pigmentado (nodular e macular) e misto não pigmentado, podendo haver crescimento radial ou vertical. Adicionalmente, referem que os melanomas orais podem exibir coloração uniforme, ou com variação de cor, sendo, na maioria dos casos, assimétricos, irregulares e ocasionalmente múltiplos. Em nossa amostra, a maioria dos casos era da forma macular pigmentada com bordas irregulares e limites mal definidos.

As modalidades de tratamento para os melanomas orais incluem ressecção cirúrgica com ou sem esvaziamento cervical, imunoterapia e radioterapia, sendo a cirurgia o tratamento de primeira escolha ${ }^{(1,11)}$. Na amostra da presente pesquisa, em três casos a imunoterapia foi o tratamento estabelecido, associando-se a radioterapia em dois casos e a cirurgia em outro. Em dois casos apenas a intervenção cirúrgica foi realizada e em um caso nenhum tratamento foi estabelecido, em virtude do óbito precoce do paciente.

Conforme relatos de Pour ${ }^{(8)}$, a maioria dos casos de melanomas orais é descoberta durante exames dentários de rotina e, infelizmente, a grande maioria dos pacientes não exibe sintomatologia nos estágios iniciais, o que acarreta uma demora de aproximadamente nove meses para a procura por atendimento, contribuindo para o atraso no diagnóstico. Esse fato pode justificar em parte alguns achados no nosso estudo, pois nos casos analisados as lesões eram bem extensas, diagnosticados em estágios avançados, conduzindo os pacientes estudados a óbito em poucos meses.

De acordo com Garcia et al. ${ }^{(2)}$ e Pour(8), o prognóstico do melanoma oral é pobre quando comparado com o do cutâneo, relatando os autores ${ }^{(8)}$ que a taxa de sobrevida de cinco anos para os pacientes com essa neoplasia não excede $5 \%$ a $9 \%$ dos casos. Dos casos que retornaram para controle em nosso estudo, todos foram a óbito bem pouco tempo depois do diagnóstico, ressaltando que em quatro dos seis casos houve disseminação da neoplasia para sítios adjacentes, alguns dos quais para cavidades e tecido ósseo, reforçando a agressividade desses tumores.

\section{Conclusão}

Com a realização desta pesquisa, detectamos que os achados no tocante ao perfil epidemiológico dos casos de melanomas cutâneos e mucosos na população estudada em muito se assemelham aos poucos estudos epidemiológicos publicados na literatura mundial, apesar de alguns dados quanto a sexo e idade em determinadas localizações anatômicas terem variado, bem como a incidência de melanomas orais na população estudada ter sido bem mais baixa do que na maioria das pesquisas avaliadas, detectando-se nesses casos pouco tempo de sobrevida após o diagnóstico, o que confirma a agressividade dos melanomas orais. As diferenças encontradas em nosso estudo podem estar relacionadas com as variações geográficas, o estilo de vida e a exposição a fatores de risco de determinadas populações. Ressalta-se que a realização de pesquisas dessa natureza é muito importante, porque contribui para o melhor conhecimento do perfil de uma determinada lesão em uma população.

\section{Referências}

1. BACHAR, G. et al. Mucosal melanomas of the head and neck: the Princess Margaret Hospital experience. Head Neck, v. 30, n. 10, p. 1325-31, 2008.

2. GARCÍA, R. G. et al. Melanoma of the oral mucosa. Clinical cases and review of the literature. Med Oral Patol Oral Cir Bucal, v. 10, p. 264-71, 2005.
3. HICKS, M. J.; FLAITZ, C. M. Oral mucosal melanoma: epidemiology and pathobiology. Oral Oncol, v. 36, n. 2, p. 152-69, 2000.

4. LASITHIOTAKIS, K. G. et al. The incidence and mortality of cutaneous melanoma in Southern Germany. Trends by anatomic site and pathologic characteristics, 1976 to 
2003. Cancer, v. 107, n. 6, p. 1331-9, 2006.

5. MELETI, M. et al. Oral malignant melanoma: review of literature. Oral Oncol, v.43, n. 2, p. 116-21, 2007.

6. PATEL, S. G. et al. Primary mucosal malignant melanoma of the head and neck. Head Neck, v. 24, p. 24757, 2002.

7. PATRICK, R. J. et al. Primary mucosal melanoma. J Am Acad Dermatol, v. 56, n. 3, p. 828-34, 2007.

8. POUR, H. Malignant melanoma of the oral cavity: a review of literature. Indian J Dent Res, v. 19, n. 1, p. 47-51, 2008.

9. PRUTHI, D. K. et al. Incidence and anatomic presentation of cutaneous malignant melanoma in central Canada during a 50-year period: 1956 to 2005. J Am Acad Dermatol, v.61, n.1, p. 44-50, 2009.

10. RIO GRANDE DO NORTE (Estado). Instituto de Defesa do Meio Ambiente (IDEMA). Aspectos físicos. Natal, 2009. Disponível em: <http://www.idema.rn.gov.br/ governo/secretarias/idema/perfilrn/Aspectos-fisicos. pdf>. Acesso em: 11 nov. 2009.

11. RAPIDIS, A. D. et al. Primary malignant melanoma of the oral mucosa. J Oral Maxillofac Surg, v. 61, p. 1132-9, 2003.

12. SORTINO-RACHOU, A. M. et al. Primary oral melanoma: population-based incidence. Oral Oncol, v. 45, p. 2548, 2009. 